\title{
Commentary: Important features of modified live virus vaccines -A comment
}

\author{
W. Jean Dodds ${ }^{1 *}$ \\ 'Hemopet, 938 Stanford Street, Santa Monica, California 90403, USA
}

Article Info

\section{Article Notes}

Received: December 24, 2017

Accepted: January 16, 2018

${ }^{*}$ Correspondence:

Dr W. Jean Dodds, Hemopet, 938 Stanford Street, Santa Monica, California 90403, USA

E-mail: info@hemopet.org

(c) 2018 Dodds WJ. This article is distributed under the terms of the Creative Commons Attribution 4.0 International License.
Prior to and since publication of my original commentary on this topic in 2014, numerous articles have appeared that address issues raised concerning vaccine safety and efficacy primarily in companion animals and humans, but also in livestock ${ }^{1-11}$. In livestock animals, earlier and recent studies have identified the adverse effects on reproduction (necrotic oophoritis, infertility, abortion and neonatal pancytopenia) from vaccinating heifers during estrus, and pregnant cows with MLV bovine herpesvirus-1 (for bovine rhinotracheitis) and bovine viral diarrhea virus ${ }^{3,8,9}$. Another study in sheep attributed the acute and chronic autoimmune/autoinflammatory (ASIA) syndrome to repetitive administration of aluminum-containing adjuvanted ovine blue tongue virus vaccines ${ }^{4}$. These issues, while mostly discussed with regard to companion animals, transcend those related to the modified live virus (MLV) vaccines discussed previously, with recent focus being on the safety of adjuvants used in killed, inactivated vaccines to accelerate, prolong, or enhance antigen-specific immune responses ${ }^{1-15}$.

While inactivated vaccines do not multiply in the host, thereby making them inherently safer than those of MLV type, they are usually less effective in initiating a complete and sustained immune protective response unless potent adjuvants are included ${ }^{6,7,13}$. Further, incorporating these adjuvants increases the risk of immediate and even delayed adverse autoimmune and inflammatory effects from the vaccine $e^{6,7,12-14}$.

Adjuvants have been used safely in human and veterinary medicine for decades, especially those containing aluminum salts and monophosphoryl lipid A in human and animal vaccines, and squalene in animal vaccines. But, these adjuvants may also induce adverse effects ${ }^{2-6,12-26}$.

A landmark review for its time addressed the vehicles used as immunomodifiers in vaccines ${ }^{16}$. Today, these include: aluminum salts, water-in-oil emulsions, biodegradable oil vehicles, oil-inwater emulsions, biodegradable microcapsules, nano- and microparticles, immune-stimulating complexes, organic oils like squalene, liposomes and lipid A, oral vaccines, and virosomes ${ }^{12-35}$. The more recent availability and popularity of sub-unit, recombinant, intranasal and oral vaccines is tempered by the fact that they also need adjuvants to elicit an optimum immune response ${ }^{13,14,16,17,28,29}$. Since then, the literature and vaccine industry has exploded with new types of adjuvants and numerous studies describing the 
safety and efficacy of adjuvants in human and animal vaccines $^{4,6,13-15,36}$. Biodegradable polymeric nanoparticles are newer vaccine vehicle adjuvants that have entrapped antigens such as proteins, peptides, or DNA. The goal of these novel adjuvants is to selectively target the host's antigen-presenting cells by controlling the release of vaccinal antigens and thus promoting the desired immune response $\mathrm{e}^{19-21,36}$. The use of dendritic cells in efficiently delivering vaccinal antigens to a host has been an important approach in vaccine technology research ${ }^{36}$. While the currently available vaccine adjuvants can successfully generate humoral antibody-mediated protection, other diseases such as tuberculosis and malaria require a cellmediated immune response for adequate protection ${ }^{17-19,22}$.

Interesting experimental findingshave shown thatgiving just two or three adjuvants simultaneously can overcome the genetic resistance to autoimmunity ${ }^{23}$. However, the dilemma emanating from concerns about vaccine safety is confounded by the fact that developmental, reproductive and whole animal toxicity studies in the target species are often excluded from the regulatory safety assessment of vaccines, because they historically have been viewed an inherently safe and non-toxic ${ }^{37,38}$. For example, MLV bovine herpesvirus vaccine is still used in pregnant cattle despite published concerns for the increased risk of infertility, abortion and neonatal pancytopenia ${ }^{8,9}$. Further, children and young animals with their more vulnerable immune system and higher risk of toxicity than adults, have the extra challenge of receiving more vaccines and adjuvants at that age $e^{8-11,26,27,37,38}$. Adjuvants also have the capacity to change individual gene expression which can adversely impact the central nervous system, brain development, and overall immune function ${ }^{23-27}$.

The ASIA autoimmune (auto-inflammatory) syndrome induced byadjuvants was first defined in 201124-26. Presently, it includes four conditions that share similar signs and symptoms and result from hyperactive immune responses: siliconosis, macrophagic myofasciitis syndrome, Gulf War syndrome and post-vaccination phenomena ${ }^{4,13,24,26}$.

Heavy metals such as the mercury and aluminum used as adjuvants and preservatives in vaccines as well as nickel, chromium, silver and gold are believed to be triggers of the ASIA syndrome ${ }^{23-27}$. The mechanism involved mainly evokes toxic and immunological reactions in the body that then present as hypersensitivity or autoimmunity. Metals can be a risk factor in a variety of autoimmune disease including autoimmune thyroiditis, multiple sclerosis, and kidney disease, and nonspecific symptoms such as chronic fatigue and myalgia ${ }^{22,24-26}$. The type of allergy induced by metals is delayed-type hypersensitivity and manifests often as a contact dermatitis ${ }^{12,22}$.

In humans, causality questions about the adverse events associated with childhood vaccines are categorized by: Can It (Potential Causality)? Did It (Retrodictive Causality)? and Will It (Predictive Causality) ${ }^{10}$. This publication from 1994, was updated in 2011 by the Institute of Medicine, National Academy of Sciences, which agreed that a causal relationship was present between some vaccines and adverse events ${ }^{10,11}$. Even though convincing evidence was presented for adverse events from mumps-measles-rubella (MMR), varicella zoster (herpes virus), influenza, hepatitis $\mathrm{B}$, meningococcal, and tetanus-containing vaccines, the group's conclusion was that "while no vaccine is 100 percent safe, very few adverse events are shown to be caused by vaccines" 11 .

A multifaceted approach is necessary to foster the recognition not only of the benefits but also of the risks of vaccines given to humans, companion animals and livestock. Further, the goal should be focused on seeking improved vaccination strategies and alternative means to prevent and control the spread of infectious diseases and their environmental impact ${ }^{3-5,8,8,11,37,38}$. As a beginning for the companion animals, the periodicity between adult booster vaccinations for the so-called "core" vaccines given to healthy individuals should be increased to three or more years, and regular monitoring of serum antibody levels (as an indirect assessment of protection against the clinically important infectious agents) should be implemented ${ }^{1,7,39,40}$.

\section{References}

1. Dodds WJ. Important features of modified live virus vaccines -- A comment. Can Vet J. 2014; 55(7): 609.

2. Dodds WJ. More bumps on the vaccine road. Adv Vet Med. 1999; 41: 715-32.

3. Deutskens F, Lamp B, Riedel CM, et al. Vaccine-induced antibodies linked to bovine neonatal pancytopenia (BNP) recognize cattle major histocompatibility complex class 1(MHC 1). Vet Res. 2011; 42: 97 107. doi:10.1186/1297-9716-42-97

4. Luján L, Pérez M, Salazar E, et al. Autoimmune/autoinflammatory syndrome induced by adjuvants (ASIA syndrome) in commercial sheep. Immunol Res. 2013; 56 (2-3): 317-324.

5. Dodds WJ. Rabies virus protection issues and therapy. Global Vaccines Immunol. 2016; 1: 51-54.

6. Dodds WJ. Adjuvants and additives in human and animal vaccines. Med Res Arch. 2016; 2(5): 1-8.

7. American Animal Hospital Association (AAHA). Canine Vaccination Task Force: Ford RB, Larson LJ, Schultz RD, Welborn LV. 2017 AAHA canine vaccination guidelines. J Am Anim Hosp Assoc 2017; October: 26-35.

8. Smith PC, Nusbaum KE, Kwapien RP, et al. Necrotic oophoritis in heifers vaccinated intravenously with infectious bovine rhinotracheitis vaccine during estrus. Am J Vet Res. 1990; 51: 969-972.

9. O’Toole D, Miller MM, Cavender JL, et al. Pathology in practice. J Am Vet Med Assoc 2012; 241: 189-191. doi.org/10.2460/javma.241.2.189

10. Stratton KR, Howe CJ, Johnston RB, Jr, eds. 1994. Institute of Medicine. In Adverse Events Associated with Childhood Vaccines: Evidence Bearing on Causality. National Academies Press, Washington, DC, 1994. 
11. Stratton K, Ford A, Rusch E, et al. eds. 2011. Committee to Review Adverse Effects of Vaccines, Institute of Medicine. In: Adverse Effects of Vaccines: Evidence and Causality. National Academies Press, Washington, DC, Aug 25, 2011.

12. Leventhal JS, Berger EM, Brauer JA, et al. Hypersensitivity reactions to vaccine constituents: a case series and review of the literature. Dermatitis. 2012; 23: 102-109.

13. Heegaard PM, Dedieu L, Johnson N, et al. Adjuvants and delivery systems in veterinary vaccinology: current state and future developments. Arch Virol. 2011; 156 (2):183-202.

14. Cerpa-Cruz S, Paredes-Casillas P, Landeros-Navarro E, et al. Adverse events following immunization with vaccines containing adjuvants. Immunol Res. 2013; 56 (2-3): 299-303.

15. Cruz-Tapias P, Agmon-Levin N, Israeli E, et al. Autoimmune (autoinflammatory) syndrome induced by adjuvants (ASIA) - animal models as a proof of concept. Curr Med Chem. 2013; 20 (32): 4030-4036.

16. Altman A, Dixon FJ. Immunomodifiers in vaccines. In Vaccine Biotechnology, eds. JL Bittle, FA Murphy, Adv Vet Sci and Comp Med. 1989; 33: 301-343: Academic Press

17. Gupta RK, Siber GR. Adjuvants for human vaccines--current status, problems and future prospects. Vaccine. 1995; 13(14): 1263-1276.

18. Spickler AR, Roth JA. Adjuvants in veterinary vaccines: modes of action and adverse effects. J Vet Intern Med. 2003; 17(3): 273-281.

19. Wilson-Welder JH, Torres MP, Kipper MJ, et al. Vaccine adjuvants: current challenges and future approaches. J Pharm Sci. 2009; 98(4): 1278-1316.

20. Kuroda Y, Nacionales DC, Akaogi J, et al. Autoimmunity induced by adjuvant hydrocarbon oil components of vaccine. Biomed Pharmacothe.r 2004; 58(5): 325-337.

21. Akagi T, Baba M, Akashi M. Biodegradable nanoparticles as vaccine adjuvants and delivery systems: regulation of immune responses by nanoparticle-based vaccine. Adv Polymer Sci. 2012; 247: 31-64.

22. Israeli E, Agmon-Levin N, Blank M, et al. Adjuvants and autoimmunity. Lupus. 2009; 18(13): 1217-1225.

23. Tomljenovic L, Shaw CA. Mechanisms of aluminum adjuvant toxicity and autoimmunity in pediatric populations. Lupus. 2012; 21(2):223-230.

24. Perricone C, Colafrancesco S, Mazor RD, et al. Autoimmune/ inflammatory syndrome induced by adjuvants (ASIA) 2013: Unveiling the pathogenic, clinical and diagnostic aspects. J Autoimmun. 2013; 47: $1-16$.

25. Shaw CA, Tomljenovic L. Aluminum in the central nervous system (CNS): toxicity in humans and animals, vaccine adjuvants, and autoimmunity. Immunol Res. 2013; 56(2-3): 304-316.
26. Stejskal V. Mercury-induced inflammation: yet another example of ASIA syndrome. Israel Med Assoc J. 2013; 15: 714-715.

27. Shaw CA, Li D, Tomljenovic L. Are there negative CNS impacts of aluminum adjuvants used in vaccines and immunotherapy? Immunotherapy. 2014; 6(10): 1055-1071.

28. Vogel FR. Immunologic adjuvants for modern vaccine formulations. Ann N Y Acad Sci. 1995; 754: 153-160.

29. Vogel FR. Improving vaccine performance with adjuvants. Clin Infect Dis. 2000; 30 Suppl 3: S266-S270.

30. Clements CJ, Griffiths E. The global impact of vaccines containing aluminum adjuvants. Vaccine. 2002; 20 Suppl 3: S24-S33.

31. Perrie Y, Mohammed AR, Kirby DJ, et al. Vaccine adjuvant systems: enhancing the efficacy of sub-unit protein antigens. Int J Pharm. 2008; 364(2): 272-280.

32. Davis HL. Novel vaccines and adjuvant systems: the utility of animal models for predicting immunogenicity in humans. Human Vaccines. 2008; 4(3): 246-250.

33. Lavelle EC, O'Hagan DT. Delivery systems and adjuvants for oral vaccines. Expert Opin Drug Deliv. 2006; 3(6): 747-762.

34. Nordly P, Madsen HB, Nielsen HM, et al. Status and future prospects of lipid-based particulate delivery systems as vaccine adjuvants and their combination with immunostimulators. Expert Opin Drug Deliv. 2009; 6(7): 657-672.

35. Richards RL, Alving CR, Wassef NM. Liposomal subunit vaccines: effects of lipid A and aluminum hydroxide on immunogenicity. J Pharm Sci. 1996; 85(12): 1286-1289.

36. Vanloubbeeck Y, Hostetter J, Jones DE. The biology of dendritic cells and their potential use in veterinary medicine. Anim Health Res Rev. 2003; 4(2): 131-142.

37. Center for Biologics Evaluation and Research, Food and Drug Administration, USDHHS, Feb 2006 Guidance for industry: Considerations for developmental toxicity studies for preventive and therapeutic vaccines for infectious disease indications, $12 \mathrm{pp}$.

38. WHO Expert Committee on Biological Standardization, World Health Organization. Guidelines on the nonclinical evaluation of vaccine adjuvants and adjuvanted vaccines. 2013. $64^{\text {th }}$ meeting, October 2125, 2013, 56 pp.

39. Dodds WJ. Vaccination protocols for dogs predisposed to vaccine reactions. J Am Anim Hosp Assoc. 2001; 38: 1-4.

40. Twark L, Dodds WJ. Clinical application of serum parvovirus and distemper virus antibody titers for determining revaccination strategies in healthy dogs. J Am Vet Med Assoc. 2000; 217: 102124. 